\title{
Géolinguistique
}

14 | 2013

Varia

\section{La compétence des noms de lieux comme clé pour lire la perception de l'espace des habitants de Villar Perosa}

The Competence of Place Names As a Key to Read the Perception of the Space of the Inhabitants of Villar Perosa

\section{Aline Pons}

\section{OpenEdition \\ Journals}

Édition électronique

URL : http://journals.openedition.org/geolinguistique/816

DOI : 10.4000/geolinguistique.816

ISSN : 2650-8176

\section{Éditeur}

UGA Éditions/Université Grenoble Alpes

\section{Édition imprimée}

Date de publication : 15 décembre 2013

Pagination : $35-56$

ISBN : 978-2-84310-264-6

ISSN : 0761-9081

\section{Référence électronique}

Aline Pons, « La compétence des noms de lieux comme clé pour lire la perception de l'espace des habitants de Villar Perosa », Géolinguistique [En ligne], 14 | 2013, mis en ligne le 30 avril 2019, consulté le 31 octobre 2020. URL : http://journals.openedition.org/geolinguistique/816 ; DOI : https://doi.org/ 10.4000/geolinguistique.816 


\title{
La compétence des noms de lieux comme clé pour lire la perception de l'espace des habitants de Villar Perosa
}

\author{
Aline Pons ${ }^{1}$ \\ Université de Turin
}

\section{Résumé}

Cet article vise à étudier la façon dont l'environnement est perçu par la communauté locale, en se fondant sur la compétence toponymique d'un échantillon de locuteurs. La méthodologie choisie pour cette étude nous a permis d'identifier certaines sous-catégories dans le corpus, en fonction à la fois des connaissances toponymiques et du partage de cette connaissance à l'intérieur de la communauté. Cela nous permet d'opérer une lecture particulière du système toponymique oral, de sorte qu'il devient possible d'avancer quelques observations sur la nature systémique du corpus ainsi que sur les relations entre la communauté et son environnement (bien que ce dernier objectif semble difficile à atteindre, le patrimoine toponymique nécessitant d'être transféré de l'oralité à la représentation cartographique). En outre, il sera possible de préciser quels endroits sont au cœur de la communauté, et lesquels sont secondaires dans la vie socio-économique du village.

\section{Mots-clés}

Toponymie, dialectes italo-romans, géolinguistique, Piémont.

1. Je remercie Giulia Sappé pour les corrections apportées au texte en français. 


\begin{abstract}
This papers aims to investigate how the environment is perceived by its own community, grounding on the toponymic competence of a sample of speakers. The methodology chosen for this study let us identify some subcategories in the corpus, depending on both the toponymic knowledge and the sharing of this knowledge inside the community. This allows for a particular reading of the oral toponymic system, so that we can make some remarks on the systemic nature of the corpus as well as on relationship between the community and its environment (although the latter goal seems harder to achieve, since the toponymic heritage must be transferred from the oral practice to a cartographic representation). Moreover, it will be possible to point out which places are central to the community, and which ones are secondary in the socio-economic life of the village.
\end{abstract}

\title{
Keywords
}

Toponymy, italo-romance dialects, geolinguistics, Piedmont.

\section{Présentation de la recherche}

Le but principal d'une recherche de toponymie est en général le relevé systématique sur le terrain, d'après la vive voix des habitants, de tous les noms de lieux employés (ou du moins vivants dans la mémoire), «che l'uomo ha dato nel corso dei secoli, per distinguerli, ai luoghi grandi e piccoli rientranti in qualche modo nella sfera dei suoi interessi »(Genre et Jalla, 1993, p. 177). Ceci est déclaré en particulier pour les travaux qui s'inscrivent dans le projet de l'Atlante Toponomastico del Piemonte Mon$\operatorname{tano}^{2}$ (ATPM).

Cet objectif a aussi guidé, en partie, cette recherche toponymique dans le village de Villar Perosa, dans lequel deux enquêtes ont été réalisées, à l'occasion de deux travaux de mémoire, en 2008 et 2011. Le deuxième travail a ouvert la recherche à toute la commune, tandis qu'en 2008 l'étude s'était limitée à une seule combe ${ }^{3}$. L'hypothèse de fond de ce travail, qui

2. Pour une présentation récente du projet, on peut faire référence à l'article de Matteo Rivoira paru dans le numéro onze de cette revue (Rivoira, 2009), ou au site <www. atpmtoponimi.it>.

3. Bien que le terme combe (ici utilisé dans le sens plus commun en France) soit, comme le disent Bessat et Germi (1993), «sans contexte le type lexical le plus représenté dans la langue française comme en francoprovençal (ALJA 118) pour désigner 
est aussi celle à la base de tout travail de toponymie, est ainsi formulée par Marrapodi : «quando si parla di repertorio toponimico ${ }^{4}$ di una comunità si parla in realtà di un'astrazione, risultante dalla somma delle singole competenze» (2006a, p. 51). Si aucun locuteur ne connaît tous les toponymes du village ${ }^{5}$ où il vit, il est intéressant, d'un côté, de mettre en lumière les «compétences individuelles» de différents informateurs, qui peuvent aider à mettre en évidence la relation entre les personnes et les lieux où elles habitent, de l'autre côté, de savoir quels noms sont universellement connus, et quels sont ceux qui restent plutôt confinés à l'usage familial ou du hameau.

\subsection{Villar Perosa}

Villar Perosa est un village d'environ 4000 habitants, situé dans la partie basse du Val Chisone, dans la province de Turin. Son territoire a été divisé par les habitants mêmes en [u 'pjay], «le plan», la région du fond de la vallée, et la pente, où il y a les hameaux historiques. Le premier cadastre disponible est de 1824 et ses cartes riches en informations de topographie et d'agronomie, montrent que le fond de la vallée se composait entièrement de terres cultivées, à l'exception de certaines zones humides ou de forêt.

La transition qui a caractérisé l'histoire de Villar Perosa, du début du $\mathrm{Xx}^{\mathrm{e}}$ siècle avec un ensemble de maisons éparses, jusqu'à la conformation actuelle du village (les maisons occupent désormais tout le fond de la vallée) prend forme avec l'installation et le développement (1907) de la nouvelle usine pour la production de roulements à billes, étroitement liée à FIAT ${ }^{6}$. L'installation de l'usine attire des ouvriers des vallées alentours et de la plaine, tandis que de nouveaux besoins apparaissent : des maisons d'ouvriers, des routes, des écoles, des installations sportives et des

les vallonnements situés entre des crêtes, des moraines longitudinales» (p. 72), il est traduit par les habitants de Villar Perosa en tant que rivière. D'autre part, une traduction similaire figure chez les habitants du proche village de Pramollo, dans les matériaux de l'ATPM (2005 [25]) comme dans ceux de l'Atlante linguistico e etnografico del Piemonte occidentale - ALEPO (Bouvier, 1985, p. 61).

4. On soulignera que cette définition rappelle celles que l'on utilise couramment pour le répertoire linguistique.

5. Les enquêtes de l'ATPM, suite à des nécessités pratiques, sont réparties en fonction des limites municipales. Nous suivrons cette répartition, bien que nous soyons conscients du fait que les limites de la compétence toponymique des locuteurs ne correspondent en aucun cas à des limites administratives (pour une réflexion sur ce sujet, voir Marrapodi, 2006a).

6. L'usine de Villar Perosa (RIV) était, elle aussi, la propriété de la famille Agnelli. 
commerces. Aujourd'hui, Villar Perosa diffère radicalement de ce qu'était le village avant l'installation de l'usine, mais a aussi changé par rapport au moment où la famille Agnelli l'a quittée. Les principales zones industrielles souffrent profondément de la crise et des entrepôts entiers ne sont plus en service. Les constructions continuent à augmenter, mais la population, au début du nouveau millénaire, est à nouveau en diminution.

Si actuellement le parler de Villar Perosa rentre dans le groupe des dialectes piémontais de type pignerolais «citoyen» (une variété du turinois), il est facile d'imaginer que, jusqu'à la fin du $\mathrm{XvI}^{\mathrm{e}}$ siècle, le village partageait avec le reste de la vallée un parler gallo-romain de type provençal-alpin.

\subsection{Méthodologie}

Les données présentées ici ont été recueillies dans deux étapes. Au cours de la première 30 personnes ont été interviewées; elles habitaient dans différentes parties du village et le but était de recueillir «tous ${ }^{7}$ » les noms de lieux que chacune d'entre elles connaissait. Les informateurs, pour la plupart âgés, ont été choisis pour leur connaissance du territoire, selon les indications de l'ATPM.

Comme dans la plupart des enquêtes menées au sein de ce projet, les interviews consistaient «en une "conversation dirigée", dont la "direction" se limite à l'illustration de la carte et au repérage pour chaque dénomination citée d'un certain nombre d'informations qui serviront à remplir la fiche qui constitue l'unité de la base de donnée» (Rivoira, 2009, p. 36).

Les 500 dénominations ainsi collectées ont été organisées en liste alphabétique, qui a ensuite été soumise à 10 informateurs parmi les 30 personnes interrogées au cours de l'étude, choisies essentiellement selon le critère de la répartition des sources à l'intérieur du territoire étudié. Chaque informateur a donc dû dire s'il connaissait, nom après nom, le lieu-dit. La question «savez-vous où est... ?» a été choisie pour deux raisons : tout d'abord pour reproduire la représentation spatiale de la compétence toponymique de chaque informateur; pour cette raison les noms qui évoquaient des histoires ou des légendes, mais que l'informateur n'a pas pu localiser dans l'espace, n'ont pas été inclus parmi les lieux-dits connus. Deuxièmement car, pour insérer les données dans un tableau Excel, nous avions besoin d'une valeur binaire $0-1$ et ne pouvions pas accepter une réponse inter-

7. Une réflexion sur la méthodologie de la récolte du matériel (top)onymique provenant de sources orales est développée par Marrapodi (2011), qui se concentre en particulier sur les stratégies permettant de dépasser les limites physiologiques de la mémoire des informateurs. 
médiaire comme : «J'ai entendu le nom mais je ne sais pas où se trouve le lieu.»

À la fin de cette deuxième étape de recherche, on obtient deux résultats intéressants : le pourcentage du corpus connu par chaque interviewé et le nombre de fois que chaque nom est reconnu dans l'échantillon des personnes interrogées.

À ces données, une autre s'ajoute, même si elle n'avait pas été recherchée intentionnellement : chaque interviewé a reconnu plus de noms que ceux qu'il avait mentionnés dans le premier entretien; également, les informateurs ont été témoins de nombreux toponymes, qui n'avaient pas encore été recueillis. Marrapodi (2011) a déjà montré à quel point la mémoire des informateurs peut être aidée par l'énumération des toponymes recueillis précédemment, mais les proportions du phénomène sont allées au-delà des attentes. La plupart des nouvelles dénominations ont été rappelées à la suite d'un nom précédemment énoncé (la récurrence des dénominations dans les systèmes onymiques populaires est connue), mais dans certains cas, la mémoire a été aidée par la construction de trajets mentaux utiles pour localiser dans l'espace le nom mentionné.

Ainsi on a pu créer, grâce à Philcarto ${ }^{8}$, des cartes qui illustrent la forme de la compétence toponymique de chaque locuteur : sur ces cartes l'emplacement géographique de chaque nom connu par l'informateur est indiqué par un point. La localisation des noms n'a pas toujours été facile : le même nom est parfois utilisé par les différents locuteurs pour des endroits relativement différents du territoire, et, comme le souligne Cusan (2013, p. 51), il n'est pas simple de «perimetrare la giusta quantità di spazio che il toponimo ritaglia dall'indefinito». Utiliser des points localisés qui indiquent l'endroit identifié par la plupart des témoins qui connaissent le nom, évite aussi la difficulté de définir les limites entre un lieu et un autre.

\section{Le corpus toponymique collecté : un matériau non homogène}

La récolte de tous les noms de lieux que les informateurs nous présentent en tant que tels, ceux en usage dans la famille proche et ceux utilisés par l'ensemble de la communauté, nous permet d'obtenir des matériaux très diversifiés, facilitant l'accès à l'une des visions possibles du paysage : si la toponymie n'est pas seulement «un mode de désignation grâce auquel il

8. Il s'agit d'un programme d'analyse statistique des données géographiques, développé par Philippe Waniez, professeur à l'université Bordeaux Segalen. Cf. <http:// philcarto.free.fr/>. 
devient possible de se repérer, de situer et dénommer précisément un lieu mais aussi une prise de possession et un moyen d'action sur lui » (Dufour, 1997, p. 188), il est intéressant d'étudier, en reprenant le titre de Dufour, qui «domestique» quel espace.

Cependant, lors de la restitution à la communauté des résultats de la recherche, il faut tenir compte, parmi les toponymes collectés, de la diffusion de chaque nom : le locuteur n'acceptera pas de voir apparaître dans la même carte (ou, dans ce cas, dans le même répertoire) un ['pra da're əd 'ka] «pré derrière maison», toponyme témoigné par un seul informateur, à côté de [la si'gota], nom d'une partie importante de la dorsale, connu par plus de la moitié des personnes interrogées. Celui qui effectue une recherche toponymique dans un village est souvent porteur d'un héritage (dans le sens indiqué par Marrapodi (2006a), en tant que somme des connaissances individuelles), qui dans son intégralité est inconnu à tout le monde : au ramasseur de champignons comme au chasseur âgé. Le chercheur a donc la responsabilité de traiter soigneusement le matériel, comme le feraient les membres de la communauté, sans mettre tous les noms de lieux dans le même $\operatorname{sac}^{9}$. La tentation du collecteur des noms de lieux peut être celle de traiter différemment les noms avec une signification opaque, qui nous semblent plus évocateurs, par rapport à ceux qui apparaissent transparents ${ }^{10}$. Celle-ci représente une tentative d'opérer une analyse indépendante des questions étymologiques, même si elles sont fascinantes et charmantes, pour se concentrer sur l'actuelle situation de diffusion et connaissance des noms. Avant de procéder à l'analyse des données recueillies, il s'avère utile de mettre en évidence comment l'exiguïté de l'échantillon rend les résultats de recherche très liés au contexte : en collaboration avec l'équipe de l'ATPM nous allons entreprendre une recherche de contrôle dans les communes de Rorà et Chiomonte, pour agrandir l'échantillon étudié.

9. Comme le dit Lurati (2004), «si sente il bisogno di muovere verso una toponimia emica, che vada al di là dell'involucro esterno e si sforzi di ricostruire gli atteggiamenti di fondo che hanno improntato le sensibilità comunitarie. È difficile, ma tanto più stimolante, accingersi a tentare di carpire al toponimo quali furono i motivi per cui una comunità assegnò un determinato nome a una zona su cui essa faticava. $\grave{E}$ l'aspetto più nuovo (non unico, evidentemente) della toponomastica moderna, ma, pure, quello più impegnativo. Anche per questo è assai poco praticato» (p. 51).

10. Même si, selon la tradition scientifique la plus consolidée de l'onomastique, la «vie sémantique des noms s'arrête dès leur formation» (Dauzat, 1960, p. 39), l'impression, dans le cas des microtoponymes oraux, est d'être immergé dans des noms qui préservent au moins une partie de signification, utile pour classer le territoire (pour une réflexion sur ce sujet, voir Rivoira, 2013). 
Les clarifications nécessaires faites, on peut supposer une classification des toponymes selon leur propagation : dans le tableau qui suit, le numéro sur la gauche indique le nombre de personnes, parmi les 10 interrogées, qui connaissent chaque nom; la définition sur la droite propose des traits communs aux noms qui présentent des valeurs de diffusion proches.
$0-3$
noms connus au niveau du voisinage et/ou obsolètes
$4-7$
$8-10$ noms connus par les habitants d'une zone du village noms connus presque universellement dans tout le village

Ces catégories seront présentées et discutées dans les paragraphes qui suivent.

\subsection{Noms connus au niveau du voisinage et/ou obsolètes}

Dans cette catégorie (que nous désignerons désormais sous l'étiquette «noms du voisinage») on recueille les noms dont la divulgation est limitée dans l'espace et le temps : dans le premier cas, il s'agit de noms se référant à de petites portions du territoire, qui répondent à l'intérêt de la famille ou des habitants des hameaux limitrophes; dans le deuxième cas il s'agit de noms qui se réfèrent à des activités disparues (anciennes mines, charbonnières), ou à des portions de terrains abandonnés, des noms qui persistent seulement dans la mémoire des personnes les plus âgées ${ }^{11}$. Les noms familiers de lieux, connus sous un nom différent dans le reste de la communauté, font également partie de la catégorie des noms du voisinage ${ }^{12}$. Dans la localité étudiée la synonymie toponymique (noms différents qui indiquent la même place) est très répandue. Nous pouvons identifier deux

11. Il manque dans ce travail, en raison du but de «sauvegarde» qui a orienté la phase de collecte, un ensemble de noms connus, seulement des jeunes générations notamment, qui se réfèrent à des commerces, des lieux de rencontre, etc. Même si ces noms ne sont pas obsolètes, nous pouvons imaginer une diffusion comparable à celle des noms inclus dans cette catégorie.

12. La multiplicité des noms pour désigner le même lieu est reconnue, et Marrapodi (2002) a essayé de décrire le phénomène dans une communauté ligurienne : «Il fatto che gli stessi luoghi possano avere nomi diversi è noto anche ai non addetti ai lavori e sembra un fatto abituale e innocuo [...] Ma qui [dans la communauté Orbasque] si tratta di un fatto diverso: le varianti sono all'interno del sistema stesso, nello stesso codice e sono in una percentuale talmente considerevole da obbligare lo studioso a rimettere in discussione l'idea di un sistema toponimico omogeneo e condiviso in egual misura da tutta la comunità: la gente può individuare con nomi diversi un numero anche elevato di siti, rendendo talora impossibile la comprensione referenziale.» (p. 291). 
causes à ce phénomène : la première est l'habitude des villaresi ${ }^{13} \mathrm{~d}$ 'indiquer un petit chalet d'alpage (ou, moins fréquemment, une parcelle de terre) avec le surnom du propriétaire, ou de l'un des composants de sa famille. En général, la famille propriétaire, ignorant le surnom qui lui est attribué, indique son chalet avec un nom différent de celui utilisé par la plupart des autres (souvent avec une cristallisation de l'appellatif par antonomase, p. ex. [la 'mjanda] «le chalet d'alpage»). La deuxième raison est le déclin des contextes dans lesquels ces noms sont socialisés. Pour donner un exemple, à une certaine époque, il était important de connaître les bornes ([i 'tcrmu]) entre les différentes parcelles, et de savoir à qui appartenait la parcelle de terrain. Aujourd'hui la propriété des terres sur le versant de la vallée est si rarement un sujet de discussion qu'une indication précise des lieux devient inutile : pour connaître les limites on fait plutôt référence au bureau technique qu'à la mémoire toponymique. Cette dernière est tellement fragmentée entre les différents foyers qu'elle suit une évolution indépendante : le résultat est que les différentes familles indiquent avec le même nom des endroits différents ou, au contraire, ils ont des noms différents pour le même lieu. Jusqu'à présent, on a toujours fait référence à des noms connus dans un contexte familial ou de hameau, mais on ne peut pas exclure la possibilité qu'un seul locuteur, pour s'orienter dans le territoire, utilise des noms de lieux connus par lui seul, pour identifier des points de repère spatiaux ${ }^{14}$ (Marrapodi (2006b, p. 49) mentionne aussi cette possibilité, en parlant des noms de lieux de la transhumance). Il faut se demander si des noms de lieux connus par une seule personne peuvent entrer pleinement dans le répertoire toponymique de la communauté. Si nous acceptons la définition de Marrapodi, selon laquelle ce répertoire est l'abstraction crée par la somme des compétences individuelles, la réponse est oui. Mais si nous croyons que le patrimoine toponymique d'une communauté devrait être utilisée au sein de la communauté elle-même, la réponse devient plus incertaine. Il est vrai que la plupart de ces noms, grâce aussi à leur création récente, sont complètement transparents, et donc compréhensibles pour les locuteurs qui partagent les points de repère de l'inventeur.

13. Ethnique utilisé par les habitants de Villar Perosa pour se désigner eux-mêmes.

14. Dans le cadre de mon travail de mémoire, je suis tombée sur un réseau de toponymes construit par un informateur pour se déplacer autour de la centrale électrique du bassin, dont son père était gardien. Dans ce cas, on peut supposer que l'informateur utilisait ces toponymes pour parler avec son père. Mais maintenant que le vieux gardien a disparu, son fils demeure le porteur unique de ce répertoire. 


\subsection{Noms connus par les habitants d'une zone du village}

À cette catégorie (que nous appellerons désormais sous l'étiquette «noms de zones ») appartiennent les noms connus par différentes familles, vivant dans des hameaux proches les uns des autres. Cette proximité ne peut être déduite à partir d'une carte, ni définie sur la base des routes d'aujourd'hui, mais doit être extrapolée de l'interprétation des compétences toponymiques des habitants. Les noms de zones sont surtout des noms qui nécessitent une bonne connaissance du territoire par les locuteurs (ce qui explique pourquoi ils sont ignorés par les habitants des hameaux éloignés) : dans cette catégorie rentrent les abris pour les outils, les charbonnières, certains petits cours d'eau, des morceaux de bois, des rochers, des fontaines et la plupart des alpages.

Il s'agissait de lieux importants pour la vie du village et qui servaient de points de repère pour le pâturage, la coupe du bois, la cueillette de champignons. Il était aussi important que ces noms soient partagés avec les habitants des villages voisins, afin d'éviter des disputes sur les frontières, mais aussi pour situer les récits de la journée.

Parmi les noms de zones il y en a qui sont connus aussi par les habitants des hameaux plus éloignés, car ils indiquent des grandes zones, que le locuteur peut facilement localiser, même sans en avoir une expérience directe : c'est le cas des noms des hameaux, des sommets, de l'île au milieu du [ki'zun] «la rivière Chisone», des ponts, des routes, des ruisseaux majeurs et des toponymes évocateurs de récits. Ces derniers sont importants pour la communauté afin d'opérer la (re)construction du paysage, et c'est pour ça qu'ils sont connus par tout le monde, même si de nombreuses personnes n'ont qu'une vague idée de leur emplacement : c'est le cas de ['roka pu'lera] «rocher des poules [?] » du [saut dəl ca'val] «saut du cheval» ou de la [ka əd le 'maske] «la maison des sorcières ${ }^{15}$ », toponymes qui sont porteurs d'une histoire partagée par toute la communauté ${ }^{16}$.

15. Mulon (1997) remarque qu' «un certain nombre de toponymes, et ce sont surtout des microtoponymes, sont de réelles mises en garde» (p. 40). Les toponymes peuvent véhiculer des interdictions, en devenant de réels panneaux d'avertissement; comme le dit Marrapodi (2006b), «la pericolosità non va intesa solo in senso fisico, ogni cultura sviluppa paure legate a credenze sul soprannaturale» (p. 55).

16. L'importance du lien entre le patrimoine toponymique et narratif de la communauté au sein des matériaux de l'ATPM a été discutée par Rivoira (2009, p. 42). 


\subsection{Noms connus presque universellement dans tout le village}

Ces noms (que nous appellerons désormais sous l'étiquette «noms communautaires ») sont connus presque universellement et désignent les principales colonies de peuplement et les points de repère de la communauté entière (de la mairie au cimetière, en passant par les écoles et les moulins), lieux dont presque tous les habitants ont une expérience directe. Dans cette catégorie il est intéressant d'analyser les cas où ces noms ne sont pas connus. La plupart des réponses négatives proviennent du témoin le plus jeune, une fille qui ne connaît pas les noms qui désignait autrefois des points de repère, mais qui ont changé de fonction de nos jours (ce qui est le cas des moulins). Chez les jeunes générations (sauf rares exceptions) l'étendue du territoire connu est très limitée, et se résume au fond de la vallée, où sont concentrées toutes les activités des villageois.

Cette classification sommaire effectuée, il est intéressant de noter que l'occurrence des noms de lieux dans le corpus n'est pas répartie de manière uniforme. Comme on peut le voir sur le graphique 1, les valeurs centrales (indiquant une distribution de zone des noms de lieux) représentent un faible pourcentage du répertoire, qui semble consister principalement de noms du voisinage d'une part, et de noms communautaires d'autre part.

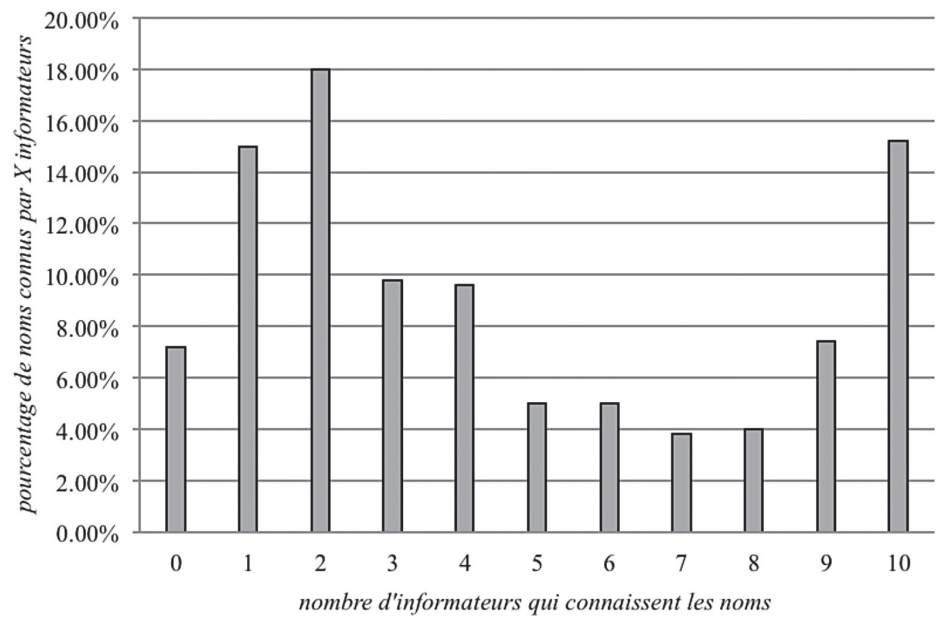

Graphique 1. - Tendance de distribution de la compétence toponymique.

Une explication de cette polarisation des compétences entre, d'une part, les noms du voisinage et, d'autre part, les noms communautaires, pourrait être le démantèlement du réseau social qui était porteur des noms de zones : les chemins des familles sont de plus en plus verticaux (du hameau 
au centre du village, jusqu'à la plaine) et de moins en moins horizontaux, entre un hameau et l'autre, et ils laissent de côté toute la zone non urbaine du village. Nous pouvons supposer qu'aujourd'hui la socialisation des noms de lieux s'effectue principalement dans le cadre de la famille immédiate, où les noms des propriétés sont transmis, avec une différenciation progressive des microtoponymes entre les familles qui n'ont pas de contacts.

À cette première socialisation s'ajoute l'expérience de vie sur le territoire, qui amène les gens à apprendre les noms des points de repère utiles pour la communication des expériences quotidiennes. Dans l'échantillon des personnes interrogées, il semble qu'il s'agit notamment des noms des hameaux et en général de ceux qui sont liés à des éléments humains, principalement concentrés dans le fond de la vallée. Il semble que le contexte social intermédiaire entre le groupe d'habitants d'un hameau et la collectivité (qui, rappelons-le, est composée d'environ 4000 personnes, même provenant de milieux très différents) soit en train de disparaître : ainsi s'affaiblit le contexte du quartier dans lequel les noms de zones ont été objet de discussion, de comparaison et d'apprentissage.

\section{L'espace de la communauté}

\subsection{Connaissance "de nom » et "de facto »}

Un autre fait qui pourrait paraître anodin, c'est que dans tous les cas étudiés la taille du territoire que l'informateur dit connaître «de facto » est supérieure à la zone définie par les noms qu'il connaît. Il est impératif à ce stade de prendre du recul et d'expliquer ce qu'on entend par «connaissance de facto » du territoire. Au cours d'un troisième cycle d'entretiens (par téléphone), nous avons demandé aux informateurs de se rappeler dans quels territoires ils avaient été et s'ils seraient en mesure d'y retourner sans se perdre. En outre, on leur a demandé s'ils connaissaient de manière égale tout le territoire dans lequel ils avaient été, ou s'il y avait des zones dont ils avaient une expérience plus approfondie. En répondant à ces questions, presque tous ont déclaré qu'ils savaient nommer une zone limitée autour de leur maison, mais qu'ils connaissaient une zone plus étendue, dans de nombreux cas (chez les informateurs âgés) équivalente à l'ensemble du territoire communal.

\subsection{La «forme» de la compétence toponymique}

Dans le cadre de cette recherche nous souhaitions, entre autres, pouvoir obtenir une représentation graphique de la «forme» de la compétence toponymique des locuteurs. Une hypothèse qui a été avancée à ce sujet 
est qu'elle se propage comme une traînée de poudre, de la maison de l'informateur aux terres voisines, avec une diminution progressive qui est en corrélation avec l'éloignement. Une autre hypothèse, plus convaincante, est que la compétence s'étend depuis la maison de l'informateur, mais que la direction de ses trajets quotidiens forme une sorte de ligne de connaissance qui unit les principaux lieux d'action du locuteur : comme l'écrit Marrapodi (2006b),

[...] parlando ancora degli informatori orbaschi, si può provare il fatto che le maggiori competenze si concentrano intorno al luogo della residenza e al luogo del lavoro seguendo percorsi individuali o di tradizione famigliare. Inoltre si possono notare una o più fasce di confine più o meno ampie a seconda del numero dei gruppi all'interno della comunità, o l'addensarsi dei toponimi in certe zone privilegiate che si dimostrano così particolarmente cariche di valore per la comunità. [...] Quali criteri di delimitazione del territorio di competenza è possibile notare in questo caso? Almeno due: il tipo di attività e il ruolo sociale [...] e il fatto che la distanza percorsa da casa propria al limite del territorio di competenza deve essere coperta a piedi in un giorno, di modo di poter tornare a casa la sera. (p. 51-52)

L'image que donnent les cartes (figure 1) ne correspond de manière complète à aucune des deux hypothèses. Dans tous les cas, nous voyons une compétence généralisée sur toute la commune, qui s'intensifie sur le fond de la vallée et autour de la zone d'intérêt de l'informateur.

En juxtaposant les cartes de divers informateurs, nous pouvons voir une certaine uniformité dans la «forme» de certaines compétences toponymiques. Alors que les deux premiers documents font état d'une compétence réduite, la troisième et la quatrième cartes sont essentiellement superposables, bien que la troisième représente une connaissance un peu plus étendue.

La cinquième et la sixième cartes ont un dessin similaire aux deux précédentes, bien que la densité de noms de lieux se soit légèrement déplacée vers le nord, pour la cinquième, et vers le sud, pour la sixième. Les quatre dernières cartes dessinent, à leur tour, des «formes» de compétence toponymiques très similaires.

Pour déterminer la compétence toponymique d'un informateur, deux variables semblent intervenir : la première, comme nous pouvions le supposer, est l'expérience personnelle, tandis que la deuxième semble être, plus que le hameau de résidence, la zone du village à laquelle l'informateur appartient. Cette distribution par zones des compétences toponymiques représente une nouveauté qui, si confirmée, pourrait détourner l'attention de la «somma delle competenze dei singoli parlanti», aux compétences 
Figure 1.

Les «formes » des compétences toponymiques de dix habitants de Villar Perosa.
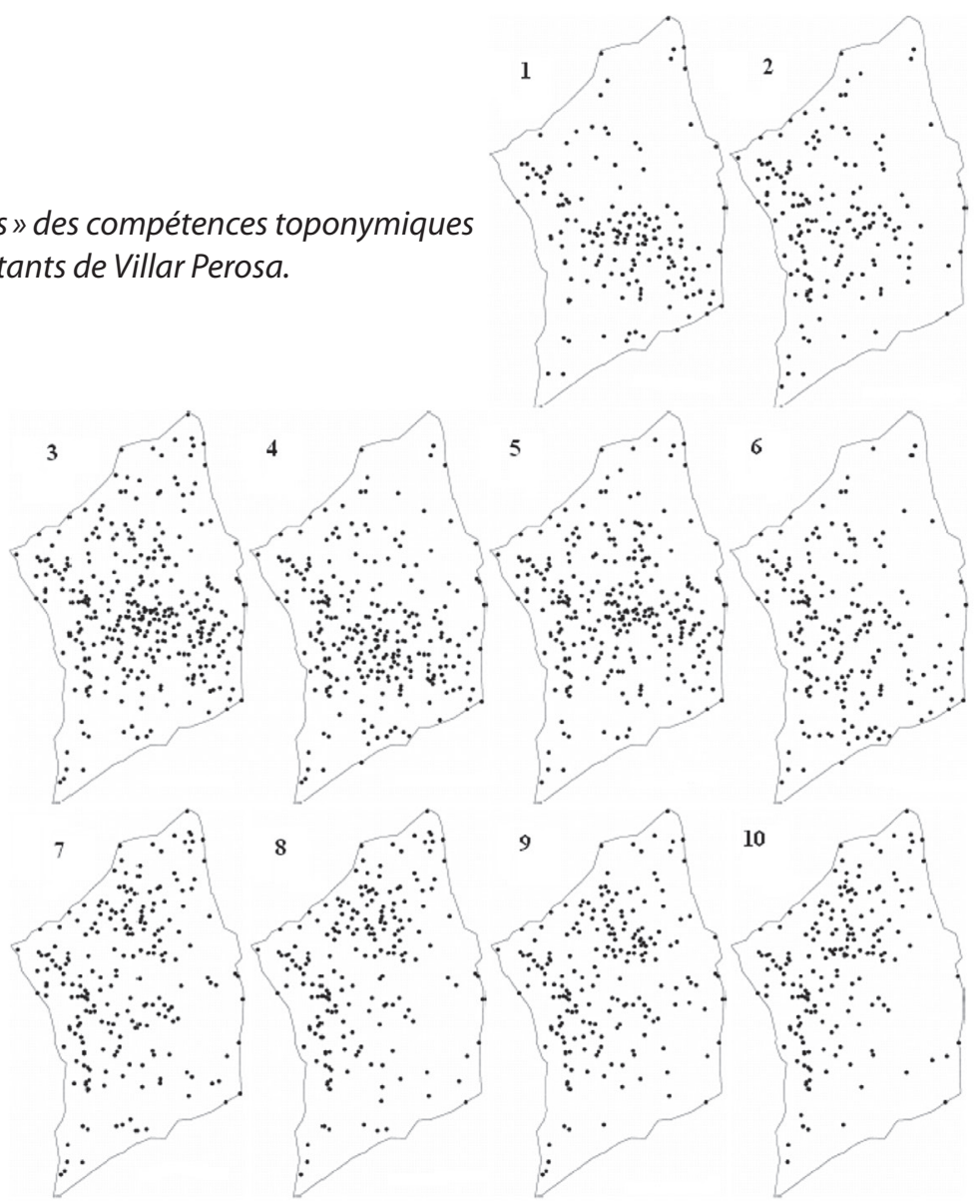

cumulées des groupes vivants dans différentes zones d'un même territoire. Ces compétences collectives pourraient être justifiées par le fait que les signes linguistiques (et donc aussi les noms de lieux) visent à communiquer le référent qu'ils indiquent, et donc impliquent un partage de code.

Bien sûr, nous ne pourrons jamais trouver deux personnes qui partagent la même compétence toponymique mais, peut-être, nous pourrions définir une «forme» prototypique de cette compétence pour chaque zone du village, autour de laquelle se développent «les formes» de chaque locuteur. En parlant de «formes», nous avons laissé de côté temporairement la distinction entre les toponymes de voisinage, de zone et de communauté, mais aussi pour les cartes qui marquent cette distinction, nous pouvons noter une substantielle uniformité de zone. 


\subsection{La diffusion des toponymes en fonction du type d'endroit désigné}

Pour approfondir l'analyse, il est intéressant, à ce stade, d'enquêter sur l'occurrence des noms en fonction de l'objet nommé. La typologie, qui est originale, a été suscitée, d'une part, par des taxonomies proposées par l'ATPM et, d'autre part, par des catégories proposées par Marrapodi (2011).

Le choix de ne pas se servir d'un modèle, qui aurait eu l'avantage de rendre les résultats comparables, a été dicté par la nécessité de prendre en compte les particularités du paysage du territoire en question : les terrains incultes et les forêts ont été combinés dans une seule macro-catégorie à cause de la difficulté d'identifier la limite entre les deux typologies environnementales, alors qu'on a essayé de séparer les prés et les champs cultivés, bien que ces derniers ne recouvrent qu'une petite partie du territoire.

Si pour les autres éléments naturels (relief et hydrographie, avec l'importante distinction entre les eaux courantes et les eaux stagnantes) on n'a pas rencontré de problèmes de classification particuliers, on ne peut pas en dire autant pour les éléments humains : on a décidé de séparer sommairement les hameaux des bâtiments individuels, en insérant dans cette dernière catégorie différents types de constructions : des maisons privées, des usines, des moulins, des magasins, etc. Une catégorie distincte pour les quartiers ${ }^{17}$ a aussi été créée.

17. La toponymie de Villar Perosa témoigne de l'existence de cinq quartiers : le [burk dəl fym] «village de la fumée», la [p'jasa] «la place» et le [burk di 'nobil] «le village des nobles» occupent le fond de la vallée, tandis que le ['karte dzur] «le quartier d'en haut» et le [kan'tuy 'rysi] «quartier de Russes» indiquent deux zones du versant, séparés par la [gray 'kumba] «la grande combe». Il n'existe aucune étude sur le sujet, mais l'impression est qu'il s'agit plutôt de noms anciens (un informateur - né en 1928 - affirme que déjà sa grand-mère utilisait ces noms de lieux), systématisés dans les années 1970, lorsque l'association de promotion touristique locale décida de les utiliser pour indiquer l'origine des chars de carnaval. Bronzat (2009, p. 85) fournit des informations intéressantes à ce sujet, extrapolées du procès verbal d'une reconnaissance cadastrale de 1701: selon ce document la communauté de Villar Perosa était divisée en quatre quartiers (avec les noms et les frontières légèrement différentes de ceux d'aujourd'hui). La division en quartiers, en dépit de l'origine en partie artificielle, est ressentie par les locuteurs (au moins par ceux des deux quartiers du versant) qui en parlant des habitants du quartier opposé disent [kui dəl kar'te da dla] «ceux du quartier d'au-delà». 


\begin{tabular}{|l|c|c|c|c|}
\hline \multicolumn{1}{|c|}{ Type d'endroit } & $\mathbf{n}^{\mathbf{0}} \mathbf{T N}$ & occurrences & \multicolumn{2}{c|}{ occurrence moyenne } \\
\hline prés et champs & 22 & 51 & 2,3 & $23 \%$ \\
\hline forêts et incultes & 198 & 497 & 2,5 & $25 \%$ \\
\hline chalets d'alpage et ruines & 44 & 141 & 3,2 & $32 \%$ \\
\hline fontaines et sources & 26 & 96 & 3,6 & $36 \%$ \\
\hline rochers, collines et sommets & 21 & 92 & 4,3 & $43 \%$ \\
\hline divers & 10 & 44 & 4,4 & $44 \%$ \\
\hline voies de communication & 29 & 144 & 4,9 & $49 \%$ \\
\hline ruisseaux & 35 & 209 & 5,6 & $56 \%$ \\
\hline quartiers & 7 & 58 & 8,3 & $83 \%$ \\
\hline lieux publics & 13 & 108 & 8,3 & $83 \%$ \\
\hline hameaux & 55 & 468 & 8,5 & $85 \%$ \\
\hline bâtiments & 40 & 348 & 8,7 & $87 \%$ \\
\hline & 500 & 2256 & & \\
\hline
\end{tabular}

Tableau 1.

Dans la première colonne sont insérés les types d'endroits plus fréquents dans la commune de Villar Perosa.

La deuxième colonne représente le nombre de toponymes dont le référent est un objet appartenant à la catégorie exprimée sur la ligne correspondante.

La troisième colonne indique le nombre d'occurrences (total) pour les toponymes appartenant à chaque catégorie de référents.

La quatrième et la cinquième colonnes montrent respectivement l'occurrence moyenne de chaque type de noms de lieux sur l'échantillon (10) et le pourcentage correspondant.

Comme on s'y attendait, les noms les plus populaires concernent les hameaux, les lieux publics et certains bâtiments qui ont une importance particulière dans la vie de la communauté (l'usine, la villa de la famille Agnelli, certains magasins). La plupart des noms recueillis, cependant, appartiennent à ces catégories de lieux dont la connaissance n'est pas partagée au sein de la communauté. Cette répartition inégale est aussi conditionnée par le type d'informateurs auquel nous nous sommes adressés sur indication de recherches de l'ATPM, selon lequel «I migliori informatori sono per lo più le persone anziane, i pastori, i cacciatori (che si rivelano preziosi per le zone alte: boschi, monti, passi) e tutti coloro che hanno trascorso la vita e svolto la loro attività sul luogo» (inédit).

Si l'on isole les toponymes communautaires, il ressort encore plus clairement que les lieux dont tous (ou presque) connaissent les noms sont ceux liés aux éléments humains : comme nous pouvons le voir dans le 
graphique 2 , les points de repère partagés, liés aux éléments naturels (fontaines, cols, forêts) sont très rares.

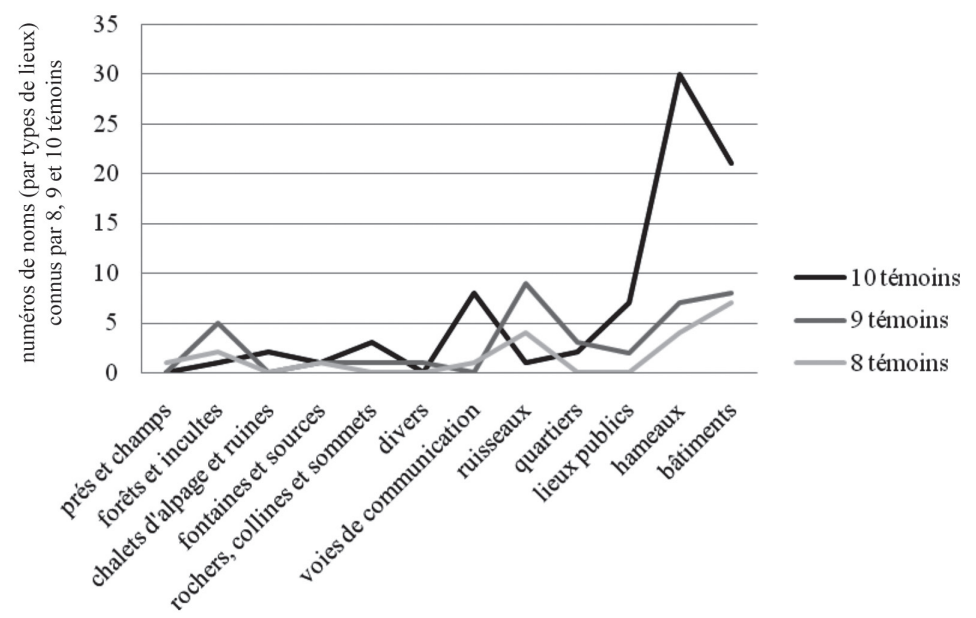

Graphique 2. - Types de lieux nommés par (presque) tout l'échantillon.

Un éclaircissement supplémentaire est nécessaire : en faisant défiler la longue liste des ruisseaux, le pourcentage de la reconnaissance des noms est particulièrement élevé; probablement cet état de fait s'explique d'abord par la transparence des toponymes (ils sont généralement composés de l'appellatif ['kumba] suivi du nom du hameau près duquel coule le ruisseau) et seulement secondairement à la connaissance réelle du cours d'eau. Certains informateurs ont facilement localisé la ['kumba du my'ret] «ruisseau des [my'rst] (nom du hameau)», sans l'avoir jamais croisé. La comparaison avec les noms des alpages est intéressante. Ceux-ci sont souvent désignés par l'appellatif ['mjanda] suivi du nom/surnom du propriétaire : dans ce cas, le taux de reconnaissance est très faible, car la localisation implique la connaissance non seulement de l'emplacement de l'alpage, mais aussi de la propriété de ce dernier.

\section{Le territoire dessiné par les noms}

Les données qui émergent jusqu'à présent de l'étude nous permettent d'essayer de construire une première esquisse de représentation du territoire, du point de vue des habitants. Cependant, plus on pénètre profondément dans la représentation de l'espace qu'ont les locuteurs, plus l'outil cartographique semble insuffisant pour décrire la microtoponymie de Villar Perosa. 
Fixer les noms de lieux sur une carte implique une détermination unique et définie du territoire nommé par un toponyme. Comme énoncé dans les paragraphes précédents, le même nom apparaît à plusieurs endroits, les frontières sont brouillées et ne coïncident ni entre les habitants de plusieurs zones, ni entre les membres d'une même famille. Nous sommes donc confrontés, au moment de la compilation d'une carte, à deux séries de problèmes.

Tout d'abord, pour avoir un nom unique (un nom = un lieu), nous devons faire un choix, souvent arbitraire, ou conditionné par le goût du collecteur, entre les différentes dénominations en usage pour le même endroit. En outre, le même nom peut être rapporté, selon différents locuteurs, à différents éléments. Cela n'arrive pas seulement avec les noms du voisinage : même la [gray 'kumba], le ruisseau qui marque la frontière entre deux quartiers du village, se trouve selon un informateur (l'aîné) dans une combe différente de celle dans laquelle elle se trouve pour tous les autres. La pluralité de noms d'un objet géographique unique n'est pas due (seulement) à la non-socialisation de certains toponymes, mais dans l'ensemble elle est aussi systémique.

Le même informateur peut aussi reconnaître quatre ou cinq noms pour ce que nous interprétons comme le même référent. Dans le cas des cours d'eau, où la situation de dénomination multiple est particulièrement vive, on peut se demander si c'était pour une raison fonctionnelle que chaque portion du ruisseau a un nom, avec l'hydronyme ['kumba] suivis par le toponyme du lieu touché par le cours d'eau : cet expédient rend la connexion des noms avec un certain tronçon du ruisseau transparente et immédiate ${ }^{18}$, et permet de savoir toujours à quelle hauteur de la rivière est l'endroit en question. Mais peut-être aussi que ces différents noms servent à raconter l'histoire du paysage, par exemple à garder «l'image» des espaces jadis consacrés au jeu de la pétanque et maintenant couverts d'immeubles, ou à nous rappeler qu'à l'endroit nommé [t fin'kwet] il y avait une pelouse.

18. Gnerre (2003), étudiant les noms des rivières de l'Amazonie, a déclaré que le nom «emerge di solito con l'uso reiterato di uno o più nomi comuni, e si rafforza con $i$ riferimenti discorsivi condivisi nell'ambito di un certo gruppo; ma mentre questo sembra essere un requisito necessario, non altrettanto necessaria sembra essere la percezione di quei nomi comuni come 'trasformati' in nomi 'propri' di quel luogo. Non solo la 'cristallizzazione' di nomi di luoghi in 'toponimi' veri e propri non è necessaria, ma a volte è del tutto ingombrante, e gli abitanti di una certa regione non sono affatto interessati a portarsi dietro, generazione dopo generazione, un fardello lessicale di nomi propri divenuti 'opachi' e quindi appresi così come il resto del lessico comune» (p. 114). 
Deuxièmement, comme mentionné auparavant $(\S 1.2)$, pour avoir des noms définis (un nom $=$ une zone délimitée par des frontières partagées) il est nécessaire de servir de médiateurs entre les différentes descriptions, qui se réfèrent souvent à des frontières tracées sur le sol, mais difficiles à détecter sur la carte (peut-être seulement à l'aide d'un GPS). Et même quand, au prix de longues promenades et de demandes répétées aux locuteurs, nous pouvons trouver sur le terrain l'endroit indiqué par le nom, sa définition n'est pas claire, et il n'est pas possible de déterminer l'endroit sinon de façon approximative : on ne veut pas affirmer que les frontières entre les zones appelées d'une manière différente n'existent pas, mais plutôt qu'elles se caractérisent comme des «zones de faiblesse» entre les différents endroits nommés et non pas comme des réalités physiquement perceptibles sur le terrain.

La difficulté d'une mise en carte d'un répertoire (micro)toponymique se rapporte probablement à la «traduction» de l'information d'un langage à l'autre : les noms de lieux se sont développés dans la langue orale et dans la pratique quotidienne du territoire, et leur transposition écrite sur une carte n'est pas sans problème. Bien sûr, il s'agit d'une étape nécessaire si nous voulons apporter à la communauté scientifique un travail de microtoponymie, mais il est fondamental de tenir compte des problèmes de la représentation cartographique.

On peut se demander si cette «réticence» des noms à la fixation est inhérente à la nature de la toponymie orale, ou si elle est un symptôme de la négligence dont sont victimes les endroits désignés. Les personnes les plus âgées n'ont que des souvenirs brumeux de lieux, peut-être lointains, où ils avaient été la dernière fois il y a des années. Dans la jeune génération (sauf de rares exceptions) l'ampleur du territoire connu (de nom et de fait) est très limitée, et même les noms de zones ou noms communautaires, qui se rapportent à des endroits élevés, évoquent des zones et rien de plus. On se demande si, lorsque les noms étaient plus largement utilisés, les zones étaient définies d'une manière unique et précise.

Il serait intéressant d'analyser, à la recherche d'une réponse par analogie, les toponymes récents, ceux qui appartiennent vraiment au patrimoine de chaque habitant de Villar Perosa (les noms liés à l'usine, ou aux lieux institutionnels) : mais cette recherche peut être trompeuse en raison de la diversité des objets nommés. Si, il y a une centaine d'années, il était vital pour la communauté de se renseigner sur les terres et les forêts, aujourd'hui on indique plutôt les places, les rues, les maisons et les lieux publics, les magasins et les infrastructures sportives, qui sont, de par leur nature, clairement définis (même si ces lieux aussi présentent souvent plus d'un nom). On pourrait supposer que, lorsque la terre avait une valeur fon- 
damentale dans la vie de la communauté, on connaissait très bien les limites de propriété, et ainsi les noms qui reflétèrent cette délimitation. Mais nous ne pouvons que le supposer : la plupart des noms recueillis indiquent des surfaces plus étendues par rapport aux parcelles cadastrales individuelles.

Ce que nous pouvons faire avec le matériel que nous avons est d'essayer de dessiner une image de ce qui devait être le territoire avant que le fond de la vallée ait été complètement urbanisé. Les noms de lieux décrivent une géographie totalement différente de celle désormais représentée sur les cartes. Certains hameaux sont devenus des routes, imbriqués dans le tissu de liens urbains, et grâce aux noms on peut distinguer les maisons, avec l'architecture ancienne, qui forment l'épine dorsale d'un village considérablement élargi. Les noms révèlent les anciens chemins, qui aujourd'hui ne sont souvent plus parcourus, après le changement dans la façon de se déplacer. Face à un village qui aujourd'hui se déplace dans une direction radiale, de la vallée aux hameaux, et vice versa, on peut imaginer des liaisons transversales qui unissaient les hameaux de la pente, et qui servaient aux enfants pour aller à l'école, aux paysans pour aller dans les champs et aux bergers pour amener les animaux au pâturage.

Une recherche toponymique dans une commune de basse vallée, comme c'est le cas de Villar Perosa, est particulièrement urgente et intéressante car elle décrit une réalité qui n'existe plus, qui est mélangée et liée à une nouvelle toponymie urbaine, très vive, qui offre des points de comparaison intéressants, et laisse puiser dans de nouveaux noms. Elle identifie ainsi dans une même zone des chemins différents et se chevauchant, qui laissent entrevoir les parcours que les noms suivent, de commun à propre, de noms connus dans un hameau à noms collectifs, de patrimoine de la communauté à patrimoine d'une génération, à lettre morte dans les archives, ou dans le mémoire d'un étudiant de passage.

Le fait qu'une recherche toponymique puisse aider à reconstruire l'histoire du paysage est bien connu. Dans le cas des données recueillies ici, cependant, nous pouvons aussi extrapoler l'image synchronique des points de repère des personnes interrogées et les localiser dans l'espace. Pour tâter le terrain dans cette direction, en attendant d'avoir l'occasion pour approfondir cet aspect, on a isolé sur la carte (carte 1) les lieux dont le nom est connu par l'ensemble de l'échantillon. On peut ainsi constater que ces noms sont concentrés dans le fond de la vallée (en particulier autour de l'usine et de la mairie) et dans les zones résidentielles, à l'exception de quelques points de repère isolés, situés comme des bornes aux extrémités de la commune. 


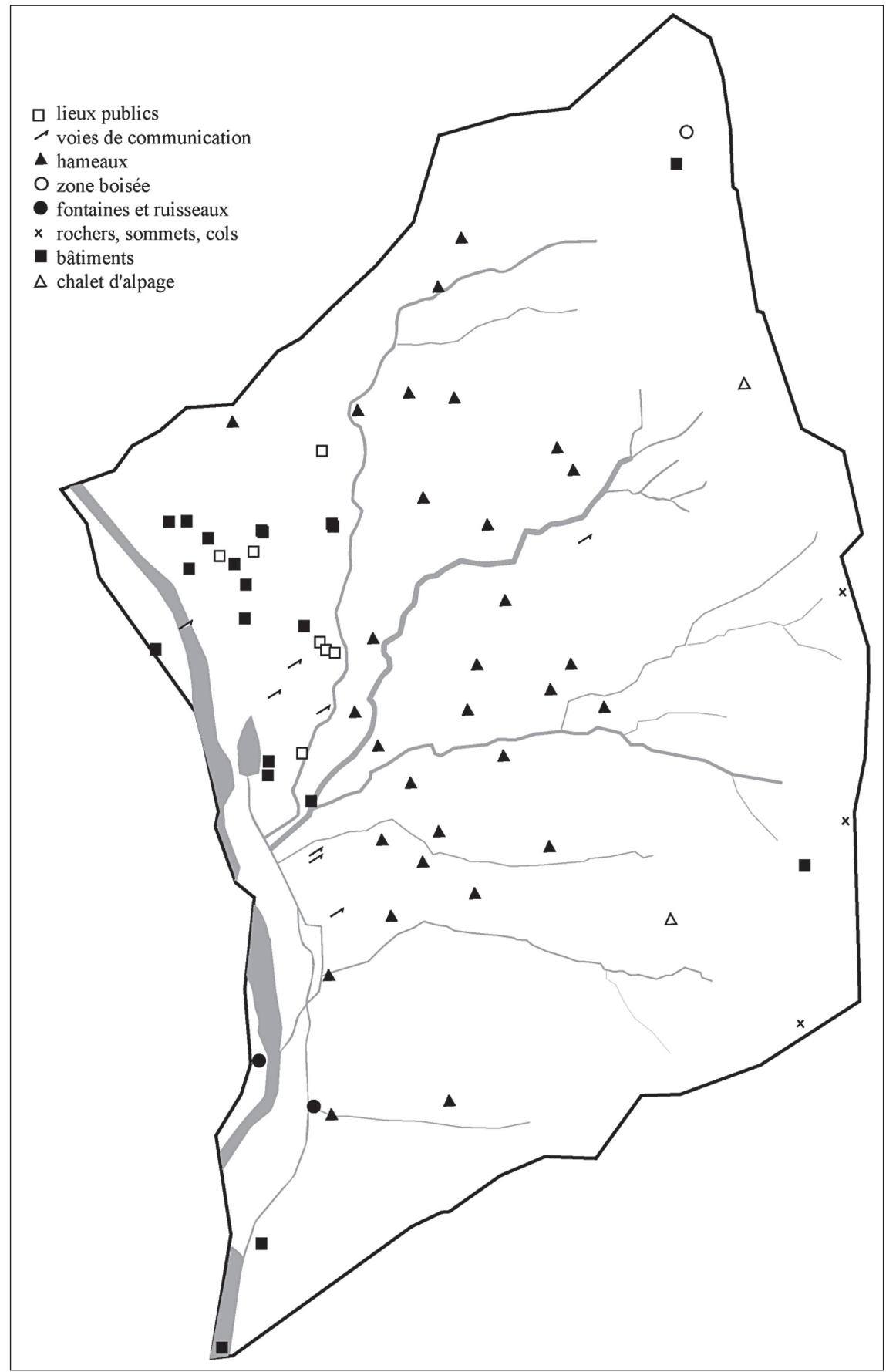

Carte 1. - Emplacement des lieux-dits connus par tous les informateurs. 


\section{RÉFÉRENCES BIBLIOGRAPHIQUES}

ATPM (Atlante Toponomastico del Piemonte Montano) : [25] Pramollo, Turin, 2005 ; Gli strumenti di lavoro (fascicule inédit); <www.atpmtoponimi.it>.

Bessat Hubert et Germi Claudette, 2001, Les noms du paysage alpin, Grenoble, Ellug.

Blanc Marina, Pascal Serenella et Pazé Piercarlo (éds), 2009, Per una storia di Villar Perosa, Comune di Villar Perosa.

Bouvier Jean-Claude, 1984, «Le ruisseau alpin. Essai d'analyse sémiolexicale des dénominations du ruisseau dans les parlers de l'Atlante linguistico e etnografico del Piemonte occidentale», dans T. Telmon et S. Canobbio (éds), Atlante linguistico e etnografico del Piemonte occidentale. Materiali e saggi, Turin, p. 53-70.

Bouvier Jean-Claude (éd.), 1997, Nommer l'espace. Le Monde alpin et rhodanien, $\mathrm{n}^{\text {os }} 2-4$.

BOuvier Jean-Claude (éd.), 2013, Le nom propre a-t-il un sens?, Actes du XVe colloque d'onomastique (Aix-en-Provence, 2010), Presses universitaires de Provence.

Bronzat Franco, 2009a, «La lingua parlata che cambia», dans M. Blanc, S. Pascal et P. Pazé (éds), Per una storia di Villar Perosa, Comune di Villar Perosa, p. 307-313.

Bronzat Franco, 2009b, «Il ripopolamento della comunità dopo l'esodo dei valdesi », dans M. Blanc, S. Pascal et P. Pazé (éds), Per una storia di Villar Perosa, Comune di Villar Perosa, p. 79-93.

Cusan Federica, 2009, «La designazione dello spazio vissuto. Analisi strutturale del sistema toponimico della comunità di Massello (Val Germanasca, Piemonte)», Bollettino dell'Atlante Linguistico Italiano, III serie, no 33, p. 97-117.

Cusan Federica, 2013, «Chabérs, Chanlasalso e Peûmian: nomi di luogo e rappresentazioni dello spazio. Riflessioni a margine dei dati raccolti dall'Atlante Toponomastico del Piemonte Montano », La Beidana, $\mathrm{n}^{\circ}$ 77, p. 44-55.

Dauzat Albert, 1960, La toponymie française, Paris, Payot.

Dufour Annie-Hélène, 1997, «Domestiquer l'espace. Quelques jalons et un exemple pour une approche ethnologique de la toponymie», dans J.-C. Bouvier (éd), Nommer l'espace. Le Monde alpin et rhodanien, $\mathrm{n}^{\text {os }}$ 2-4, p. 187-200.

FINCO Franco (éd.), 2007, Atti del secondo convegno sulla toponomastica friulana, Udine, Società Filolofica Friulana.

Frau Giovanni (éd.), 1990, Atti del primo convegno sulla toponomastica friulana, Udine, Società Filologica Friulana.

GenRe Arturo et Jalla Daniele, 1990, «Il progetto di ricerca sulla toponomastica del Piemonte Montano», dans G. Frau (éd.), Atti del primo convegno sulla toponomastica friulana, Udine, Società Filologica Friulana, p. 117-128.

Gnerre Maurizio, 2003, La saggezza dei fiumi, Rome, Meltemi.

Lurati Ottavio, 2004, In Lombardia e in Ticino. Storia dei nomi di luogo, Florence, Cesati. 
MARRAPODI Giorgio, 2002, «Varianti toponimiche, toponimi criptolalici, funzione disorientativa del TN nei sistemi toponimici popolari», Quaderni di Semantica, vol. XXIII, n ${ }^{\circ} 2$, p. 17-44.

MARRAPODI Giorgio, 2006a, «Il concetto di confine nella ricerca toponomastica», Rivista Italiana di Onomastica, vol. 12, n 1, p. 43-63.

MARRAPODI Giorgio, 2006b, Teoria e prassi dei sistemi onimici popolari: la comunità orbasca (Appennino Ligure centrale) e i suoi nomi propri, Quaderni della Rivista Italiana di Onomastica, Rome, Società editrice romana.

MARRAPOdi Giorgio, 2007, «Tassonomia dei sistemi toponimici popolari: individualità del TN e ricorsività lessicale», dans F. Finco (éd.), Atti del secondo convegno sulla toponomastica friulana, Udine, Società Filolofica Friulana, p. 259-273.

MARRAPODI Giorgio, 2011, «Metodologia delle interviste e strategia di raccolta dati in (top)onomastica», Rivista Italiana di Onomastica, vol. 17, n 2, p. 503515.

Mulon Marianne, 1997, «Les noms de la peur, la peur des noms », dans J.-C. Bouvier (éd.), Nommer l'espace. Le Monde alpin et rhodanien, $\mathrm{n}^{\text {os }} 2-4$, p. 39-45.

PhilcARTo, <http://philcarto.free.fr/>.

Pons Aline, 2011, La toponomastica di Villar Perosa. Patrimonio della comunità e competenza dei singoli parlanti, Mémoire, Università degli Studi di Torino.

Rivoira Matteo, 2009, «L'Atlante Toponomastico del Piemonte Montano (ATPM) : principes, méthodes et résultats », Géolinguistique, $\mathrm{n}^{\circ} 11$, Centre de dialectologie, Université de Grenoble, p. 29-49.

Rivoira Matteo, 2013, «Système onymique et signification. Le cas de la Coumba dî Charbounî dans la Vallée du Pélis (Piémont)», dans J.-C. Bouvier (éd.), Le nom propre a-t-il un sens?, Actes du XVe colloque d'onomastique (Aix-enProvence, 2010), Presses universitaires de Provence, p. 93-104.

Telmon Tullio et Canobbio Sabina (éds), 1984, Atlante linguistico ed etnografico del Piemonte occidentale (ALEPO). Materiali e saggi, Turin. 